\title{
Configuración y restricción de los derechos subjetivos a partir de un análisis de las posiciones jurídicas fundamentales en juego*
}

\section{María Claudia Mercio Cachapuz}

Resumen: El presente estudio se ocupa de uno de los temas centrales para la teoría general del derecho, la filosofía del derecho y el derecho civil. Se trata del análisis del contenido, configuración y restricción de los derechos subjetivos. El trabajo se propone el estudio del problema de las limitaciones y de la ponderación de los derechos subjetivos, tanto en una dimensión institucional como en una relación concreta de conflicto de intereses. Se comparan las visiones de Häberle y de Alexy con relación a la adopción de las teorías interna y externa de las restricciones de los derechos fundamentales, para proponer, a la luz del modelo analítico de posiciones jurídicas fundamentales de Hohfeld, el análisis de casos judiciales complejos.

Palabras clave: Teoría general del derecho, derechos subjetivos, argumentación, interpretación, restricción de derechos.

Fecha de recepción: 25 de julio de 20r6. Fecha de aceptación: 8 de mayo de 2017. Para citar el artículo: Mercio Cachapuz, M. C., "Configuración y restricción de los derechos subjetivos a partir de un análisis de las posiciones jurídicas fundamentales en juego", Revista de Derecho Privado, Universidad Externado de Colombia, n. $^{\circ} 33$, julio-diciembre de 2017,5 I-72.

DOI: https://doi.org/I0.18601/or 234366.n33.03

** Doctora en Derecho Civil, Universidad Federal de Rio Grande do Sul, Brasil. Profesora de Derecho Civil en la Universidad Federal de Rio Grande do Sul (Ufrgs), Brasil, y de la maestría en Derecho y Sociedad de Unilasalle, Brasil. Magistrada con actuación en la Corte de Apelación en el Tribunal de Justicia de Rio Grande do Sul, Brasil. Contacto: MCMCachapuz@ti.rs.gov.br 


\title{
The Limits of Subjective Rights through a Model of Basic Legal Positions
}

\begin{abstract}
Aвstract: The aim of this paper is to study the problem of the limits of constitutional rights, in an institutional level or in a concrete situation of conflict of interest. The paper proposes a comparison of Häberle's and Alexy's point of view, regarding the adoption of internal and external theories of restrictions on rights. It also proposes, through the Hohfeld's analytical model of basic legal positions, the correct interpretation to the analysis of hard cases.
\end{abstract}

KEywords: General Legal Theory, subjective rights, argumentation, interpretation, limits of rights.

\section{Introducción}

Uno de los temas centrales que es preciso enfrentar cuando se analizan los derechos subjetivos en el ámbito interdisciplinario de la teoría general del derecho y de los conceptos fundamentales de derecho civil es, sin duda, el relacionado con la configuración y la restricción de los derechos ${ }^{\mathrm{I}}$. Se parte de la constatación de que las normas iusfundamentales irradian el derecho civil ${ }^{2}$. En este contexto, no es posible desvincular el estudio de los institutos del derecho civil, y de todas las relaciones que lo integran, del ámbito de actuación de los derechos fundamentales3.

I La idea fundamental, según Alexy, es que "los derechos están sujetos a restricciones y pueden ser delimitados o limitados" (Alexy, R., Teoría de los derechos fundamentales, Madrid, CEPc, 200 I, 267). El problema jurídico, por lo tanto, no está relacionado con el concepto de restricción, sino con el alcance de su manifestación: "Estos [problemas] resultan exclusivamente de la determinación del contenido y alcance permitido de las restricciones como así también de la distinción entre restricción, por una parte, y cosas tales como regulaciones, configuraciones y concreciones, por otra" (ibid., 267). Para Alexy, la distinción esencial es entre normas restrictivas y no restrictivas de los derechos, justamente porque implican consecuencias para la justificación iusfundamental: "Una norma no restrictiva en el ámbito de un derecho fundamental es una norma que tiene algo que ver con aquello que abarca el derecho fundamental. Siguiendo una terminología difundida, puede decirse que esta norma lo configura" (ibid., 322).

2 En la doctrina de Alexy, esto encuentra relación con la propia idea de derecho fundamental "como un todo": "Un derecho fundamental como un todo es un haz de posiciones iusfundamentales", que corresponde a "la adscripción de un haz de normas a una disposición de derecho fundamental" (ibid., 24I).

3 El análisis distintivo aparece en la doctrina de H. C. Nipperdey, con la interpretación del artículo 2.I de la Constitución Federal alemana: "O artigo 2.I contém direito imediatamente vigente no sentido do artigo I.III, contudo vincula ele não somente o poder estatal, mas também cada paticular imediatamente [...]. Ele contém, portanto, não somente um direito constitucional público-subjetivo, mas também direitos, constitucionalmente documentados, de cada pessoa contra cada outra, isto é, portanto, um direito privado subjetivo" (Nipperdey, H. C. "Livre desenvolvimento da personalidade", en Dürig, G., Nipperdey, H. C. y Schwabe, J. Direitos fundamentais e direito privado. Textos clásicos, Porto Alegre, Sérgio Antônio Fabris Editor, 201 2, 76). 
La idea es que las relaciones iusfundamentales pueden estar asociadas tanto a una dimensión universal de establecimiento de institutos jurídico-civiles, mediante la construcción de enunciados dogmáticos (normativos, jurisprudenciales, doctrinarios, argumentativos), como a una dimensión particular de apropiación de los efectos jurídicos en el ámbito de las relaciones civiles entre los individuos. Häberle sistematizó esta dualidad de los derechos fundamentales, reconociendo una dimensión institucional con alcance universal a partir de perspectivas de libertad, y una dimensión jurídico-individual de esos mismos derechos cuando son comprendidos, en su definición, como "derechos de la persona" 4 . El alcance de ese "doble carácter de los derechos fundamentales" 5 , en la visión de Alexy", permite que se extraiga una comprensión igualmente dual con relación a las restricciones a los derechos fundamentales y, por extensión, a la irradiación de efectos hacia los derechos subjetivo-civiles7. Una polémica que, para Alexy, no se traduce en una mera cuestión de constitución conceptual dual -entre lo que sea hipótesis de aplicación de una teoría interna o de una teoría externa de restricción a los derechos ${ }^{8}$-, sino que demuestra las íntimas conexiones entre restricciones y presupuestos normativos generales.

4 Häвerle, P., La garantía del contenido esencial de los derechos fundamentales, Madrid, Dykinson, 2003, 73. Según Häberle, "los derechos fundamentales de la Ley Fundamental tienen un doble contenido jurídico-constitucional. Por un lado, presentan una dimensión jurídico-individual; garantizan a sus titulares un derecho público subjetivo; son 'derechos de la persona'. [...] Por otro lado, están caracterizados por una 'dimensión institucional'. Implican la garantía jurídicoconstitucional de ámbitos vitales regulados y conformados con arreglo a criterios de libertad" (2003, 73).

$5 \quad$ Ibid., 73 .

6 Ver Alexy, Teoría de los derechos fundamentales, cit., 322: "Quien más lejos llega es Häberle. Según él, todos los derechos fundamentales son 'susceptibles y requieren' no sólo una restricción legal sino también una 'configuración legal'. Häberle no sólo extiende el ámbito de la configuración a todos los derechos fundamentales, sino que utiliza también un concepto muy amplio de configuración. [...] No habría nada que objetar en contra de una tal concepción amplia del concepto de configuración como concepto superior, tanto para la actividad restrictiva como no restrictiva del legislador. Pero, mientras juegue (también) un papel como concepto opuesto al de restricción (limitación), hay razones para criticarlo. Cuando algo es restrictivo desde algún punto de vista, sigue siendo restrictivo, aun cuando desde algún otro punto de vista sea configurador" (ibid., 323 ).

7 Según Alexy, "la cuestión de saber si y en qué medida las así fundamentadas restricciones de la libertad jurídica general deben ser consideradas como restricciones de derechos fundamentales conduce" (también) "al problema del llamado efecto horizontal o en terceros de los derechos fundamentales" (ibid., 325 ).

8 Siguiendo a Alexy, la distinción entre teoría externa e interna de restricciones a derechos fundamentales tiene relación con el concepto de derecho y sus restricciones: "Según la teoría externa, no existe ninguna relación necesaria entre el concepto de derecho y el de restricción. La relación es creada sólo a través de una necesidad externa al derecho, de compatibilizar los derechos de diferentes individuos como así también los derechos individuales y los bienes colectivos. Una imagen totalmente distinta subyace a la llamada teoría interna. Según ella, no existen dos cosas, el derecho y sus restricciones, sino sólo una: el derecho con un determinado contenido. El concepto de restricción es sustituido por el de límite” (ibid., 268-269). 
De acuerdo con Alexy, "quien sostiene una teoría individualista del Estado y la sociedad, tenderá más a la teoría externa, y aquel a quien le interese la posición de miembro o membresía en una comunidad, tenderá a la teoría interna"9. La explicación de tal afirmación, sin embargo, no es de carácter político-jurídico, aunque pueda permitir una asociación entre el acto de interpretar las restricciones a los derechos subjetivos y visiones ideológicas distintas sobre cuestiones de libertad y justicia. La preocupación de Alexy está centrada en la posible asociación entre la restricción de un derecho y la situación de configuración de ese mismo derecho -sea por parte del legislador, sea por parte del intérprete para el caso- ${ }^{10}$.

Es verdad que la preocupación de Häberle está centrada en la actividad del legislador y no, de una forma específica, en la del intérprete. Pero sus conclusiones en términos legislativos condicionan la actividad de interpretación, en la medida en que se pueda considerar que toda la interpretación en abstracto también condiciona la interpretación en concreto ${ }^{\mathrm{II}}$. De allí que este estudio parta del examen de la extensión del concepto de restricción (lato sensu) aplicado por el intérprete ${ }^{\mathrm{I} 2}$, $\mathrm{y}$ de la medida en la que tal aplicación corresponde a las exigencias de una racionalidad suficiente para la justificación de las restricciones adoptadas.

$9 \quad$ Ibid., 269.

io Como explica el autor, "la corrección de la teoría externa o de la interna depende, esencialmente, del hecho de que las normas iusfundamentales sean consideradas como reglas o principios y las posiciones iusfundamentales, como posiciones definitivas o prima facie. Si se parte de posiciones definitivas, es posible refutar la teoría externa; si se parte de posiciones prima facie, la teoría interna" (ibid., 269).

I I La distinción ofrecida es sustentada por Guastini, para quien hay una separación dual en la idea de interpretación jurídica, como la interpretación en abstracto y la interpretación en concreto (Guastini, R., Distinguiendo. Estudios de teoría y metateoría del derecho, Barcelona, Gedisa, I999). Como explica Linfante Vidal, "en el primer caso [de la interpretación en abstracto] se trataría de identificar el contenido del significado - esto es, el contenido normativo (la norma o las normas)- expresado por y/o lógicamente implícito en un texto normativo, y ello sin hacer referencia a ningún caso concreto. En el caso de la interpretación 'en concreto' se trataría, sin embargo, de subsumir un caso concreto en el campo de aplicación de una norma previamente identificada 'en abstracto"” (VIDAL, I. L., "Interpretación y aplicación del derecho", en LAGIER, D. G. (coord.), Conceptos básicos del derecho, Madrid, Marcial Pons, 20 I 5, I 93). Hay, por supuesto, una distinción lógica entre los dos momentos de interpretación, como sustenta Lifante Vidal con respecto a la doctrina de Alexy: "Los argumentos que expresan una vinculación al tenor literal de la ley o a la voluntad del legislador histórico prevalecen sobre otros argumentos, a no ser que puedan aducirse motivos racionales que concedan prioridad a otros argumentos" (ibid., I98). Como distingue Linfante Vidal, "podemos considerar que aunque el significado literal, y en algunos casos quizás también la intención del legislador, juegan un papel relevante en la interpretación jurídica, son el punto de partida, y en algunas ocasiones también pueden ser el de llegada, pero el proceso interpretativo implica atender razones de por qué interpretar en un cierto sentido, razones que no pueden ser el mero dato objetivo de un significado literal o de una determinada intención” (ibid., r99).

I La expresión "intérprete jurídico" se entiende bajo una idea amplia que se refiere tanto a la actividad del intérprete en abstracto -legislador- como a la actividad del intérprete en concreto -la actividad del juez o del doctrinante-. 
A partir del examen de las diferencias entre las teorías de restricciones de derechos en Alexy y Häberle, se estudia la posibilidad de restringir los derechos subjetivos y justificar la medida de esta restricción. Al mismo tiempo, la investigación se relaciona con la propuesta de W. N. Hohfeld, del establecimiento de tablas de conceptos jurídicos fundamentales ${ }^{13}$, que agrupa en dos conjuntos distintos -uno de correlativos y otro de opuestos ${ }^{\mathrm{I}}$ - situaciones de tipo muy diverso en términos jurídicos.

\section{Los derechos fundamentales en Häberle}

Häberle, al preocuparse por la discusión normativa sobre la garantía de un contenido esencial de los derechos fundamentales, defiende la tesis de que los derechos fundamentales comparten un doble carácter en su estructura. O sea, estos derechos presentan, de un lado, una dimensión institucional-objetiva, en la que estaría contenida una "garantía jurídico-constitucional de ámbitos vitales regulados y conformados con arreglo a criterios de libertad" ${ }^{15}$; y, de otro lado, una dimensión jurídico-individual, en la que se garantiza a los titulares (individuales o colectivos) derechos públicos subjetivos. Se trata de dimensiones complementarias que, según Häberle, no se contraponen ni se corresponden en una relación de medio a fin, sino que, más bien, se ven integradas en una simultaneidad de actuación en términos interpretativos: "En este enfoque de los derechos fundamentales se toma en consideración tanto lo 'inevitablemente institucional' en el Derecho como lo personal" ${ }^{16}$.

I 3 En la apreciación de Genaro Carrió, "Hohfeld cumpre su tarea mediante el examen de una abundante cantidad de usos vigentes, tomados de fallos judiciales y de obras teóricas de juristas. Sobre esa base aísla ocho conceptos jurídicos fundamentales, que agrupa en dos tablas, una de correlativos y otra de opuestos. Ambas tienen los mismos ingredientes, sólo que agrupados con criterios distintos" (Carrió, Genaro, "Nota preliminar", en Hohfeld, W. N., Conceptos jurídicos fundamentales, México, Fontamara, 2009, I 5 ).

I4 Siguiendo a Hohfeld, "en la tabla de los correlativos, se destacan las cuatro modalidades activas -que suelen ser encubiertas por un uso indiscriminado de la palabra 'derecho' (subjetivo)- y se las presenta en su relación con las cuatro modalidades pasivas correspondientes. Mientras que cada una de las modalidades activas identifica la situación de un sujeto A en su relación con un sujeto B, cada una de las modalidades pasivas identifica la situación que, en cada caso, tiene B frente a A. [...] Distinta es la situación que exhibe la tabla de los opuestos. Aquí cada modalidad jurídica activa es presentada en conexión con aquella modalidad pasiva que, en lugar de complementarla, se le opone como su contradictoria en cabeza del mismo sujeto" (CARrió, Genaro. "Nota preliminar", cit., I 5).

I 5 Häвerle, P., La garantía del contenido esencial de los derechos fundamentales, Madrid, Dykinson, 2003, 73 .

I6 Ibid., 75. En la visión del autor, la dimensión institucional de los derechos fundamentales y la dimensión jurídico-individual deben ser entendidas a partir de "una relación recíproca y de paridad jerárquica" (ibid., 74). 
Este condicionamiento recíproco de las dos dimensiones que componen, según Häberle, la esencia de los derechos fundamentales ${ }^{17}$ debe seguir determinado por una pretensión de promoción de la libertad. En más de una oportunidad, Häberle acentúa que el reconocimiento de una doble dimensión a los derechos fundamentales no rechaza la promoción de un sentido de libertad de las relaciones iusfundamentales ${ }^{\mathrm{I}}$, incluso cuando se trata de establecer un carácter institucional. "El objetivo del pensamiento institucional es, por lo tanto, insertar al individuo y su libertad en las regulaciones y relaciones vitales objetivas y, al revés, llevar a estas regulaciones la creatividad y la potencia creadora de los individuos" $"$.

La libertad que se considera como orientadora del establecimiento de derechos fundamentales no tiene rasgos de un pensamiento voluntarista ${ }^{20}$. Tampoco debe seguir, para Häberle, una visión formalista o positivista del derecho ${ }^{2 \mathrm{I}}$. La dimensión institucional-objetiva estructurada pretende ser justificada por una orientación de interpretación que promueva la conexión entre el proceso de recepción de derechos fundamentales en el ámbito de una constitución y la propia identificación de esos derechos en la sociedad: "Por medio de estas normas jurídicas, que no son mera ‘transformación' de la libertad o de los derechos fundamentales, se hace efectiva la idea de derechos fundamentales en la realidad social"22. El ámbito institucional es complementado por la dimensión subjetiva de los derechos fundamentales, ofrecida por la actuación del individuo en concreto al llenar "de vida" los institutos jurídicos ${ }^{23}$.

Si existe una identificación simultánea de las dimensiones pertinentes a los derechos fundamentales, de forma que una esté esencialmente vinculada a la otra en la propia configuración de los derechos, la lógica en Häberle es que toda y cualquier restricción a los derechos fundamentales esté necesariamente relacionada con una concepción amplia de configuración de esos mismos derechos. Häberle llega a sostener que, “entre otras cosas, es característico de la dimensión

I 7 Ibid., 74 .

I 8 Conforme al autor, "la institucionalización de los derechos fundamentales no va paralela a una difuminación de la libertad individual; al contrario, pretende y opera un fortalecimiento de la libertad" (HäвERle, P., op. cit., 75).

I9 Ibid., 82 .

20 Según Häberle, "el voluntarismo reduce el Derecho a las relaciones entre voluntades" (ibid., 88).

2 I Sostiene Häberle: "El positivismo y el formalismo ignoran la relación con la realidad del derecho y los derechos fundamentales. Su validez fáctica -y esta es esencialmente un fenómeno institucional- no tiene ningún tipo de relevancia para este pensamiento" (ibid., 97). En la discusión filosófica, según Moreso y Vilajosana, no se trata, de otra parte, de distinguir y clasificar las distintas concepciones del derecho, sino de ofrecer el "análisis crítico de los argumentos que son usados en la defensa de las respectivas tesis" (Moreso, J. J. y vilajosana, J. M., Introducción a la teoría del derecho, Madrid, Marcial Pons, 2004, I92).

22 HäвERle, P., op. cit., 97.

23 Ibid., 99. 
institucional de los derechos fundamentales que no se trata de espacios jurídicamente vacíos, sino de ámbitos vitales jurídicamente conformados" ${ }^{24}$.

Lo contrario ocurre en la doctrina de Alexy, que señala que el simple hecho de que haya una norma que desempeñe una función restrictiva del derecho de alguien no otorga, simultáneamente, carácter de configuración o de conformidad a ese mismo derecho ${ }^{25}$. En la perspectiva de promoción de la libertad, la norma que restringe la conducta -sea en la definición del tipo penal, sea en el establecimiento de una determinada prohibición u orden administrativo o civiles siempre restrictiva, y no corresponde presuponer, por su establecimiento, un acto de configuración de un derecho.

Al sostener que la función del legislador es la de limitación y de conformación de los derechos fundamentales -refiriéndose de forma específica no apenas a normas que establezcan competencias, sino, principalmente, a normas que garanticen derechos fundamentales-, Häberle retoma el parámetro de la dualidad para reconocer un doble contenido a la garantía de los derechos fundamentales. Aclara que, por parte del legislador, hay un condicionamiento que implica la prohibición de lesionar un derecho fundamental y una determinación dirigida a la conformación específica de cada derecho ${ }^{26}$. Corresponde conferir a la hipótesis de configuración un carácter aún más amplio que a la de restricción, en la medida en que la actividad de restricción se disuelve en la idea de existencia de un límite inmanente a la conformación de cualquier derecho. Y este es un punto central en la doctrina de Häberle para el establecimiento de la interpretación en abstracto.

El problema es que tal comprensión jurídica conduce a la imposibilidad de sustentación de la relación de dualidad pretendida por el autor. Para Häberle, siempre hay configuración de derechos, siendo parámetros de conformación del actuar humano a un "ideal" destacado en el ámbito constitucional. En el ámbito ordinario, dados los límites superiores establecidos en la constitución, la actividad legislativa pasa a ser discrecional. Algo que, por lo tanto, no se exime de la crítica sostenida por el propio autor, en cuanto a una construcción de justificación eminentemente formalista en materia de restricciones de los derechos fundamentales ${ }^{27}$. "De 'conformación' puede conceptualmente hablarse

24 Ibid., I 15.

25 Según Alexy, "Häberle no sólo extiende el ámbito de la configuración a todos los derechos fundamentales, sino que utiliza también un concepto muy amplio de configuración". Citando a Häberle, refiere que "en la configuración, de lo que se trata es 'del objetivo de la Constitución, de la realización de los derechos fundamentales en la vida social"” (Alexy, R., Teoría de los derechos fundamentales, Madrid, CEPC, 2001,322$).$

26 Häberle, P., op. cit., I69.

27 La crítica no consigue ser apartada ni siquiera cuando Häberle explica que, conceptualmente, no se debe hablar de discrecionalidad cuando la actividad del legislador está pautada por una doble dimensión de garantía a los derechos fundamentales: "Conceptualmente también le es retirada al legislador la discrecionalidad, dado que se habla de la función de limitación y de con- 
solo donde existe algo a conformar: es el 'ideal' que la Constitución ha elaborado de cada derecho fundamental. En lo restante, la Constitución se despojó de su propio rango, abrió al legislador el camino para la regulación normativa de tipo discrecional" 28 .

La explicación de Häberle acerca del problema de la conformación de derechos de forma amplia igualmente no se aparta del riesgo apuntado a un voluntarismo en términos jurídicos. Ello se observa justamente por la dificultad del autor para sustentar cómo debe ocurrir la intervención del legislador en términos de conformación de derechos por la identificación de determinados ideales sociales $^{29}$.

De "conformación” se habla aquí con toda intención: la legislación da al derecho la forma concreta, se trata de una actividad jurídica creativa de alto rango. La intervención del legislador en el ámbito de los derechos fundamentales es tanto más apremiante, y tanto mayor por consiguiente la necesidad de conformación y limitación de un derecho de libertad, cuanto más intensa sea la "relación social" de la correspondiente libertad. Puesto que la libertad de creencias opera en el interior de la persona, la función de conformación y limitación del legislador pasa claramente a segundo término. Es, por ello, la libertad menos "social". No obstante, tan pronto como una libertad entra en "contacto social" -y este es el caso normal-, y la libertad iusfundamental precisamente trata en lo esencial de la coordinación de ámbitos vitales, será accesible al derecho y a la regulación del legislador ordinario. El legislador se ve entonces inducido tanto a la conformación como a la limitación de los derechos fundamentales ${ }^{\circ}$.

Häberle, aun al tratar de la naturaleza y de la función de legislar en materia de derechos fundamentales, llega a afirmar, como premisa y sin advertir de que se trata de un argumento meramente conceptual, que "el contenido de la Constitución no está en ningún caso amenazado por uno de tales procesos de concreción; al contrario, experimenta una reafirmación" ${ }^{3}$. Pero se podría sostener que no hay desafío jurídico al enunciado dogmático, en términos de progreso ${ }^{32}$, cuando

formación. La cuestión sobre la función del legislador es, así pues, al mismo tiempo la cuestión sobre los límites (internos) de su actividad" (HäвERLe, P., op. cit., I69). Tratándose del ejercicio efectivo de competencias por parte del legislador, no se puede justificar la discrecionalidad con bases eminentemente conceptuales, como es referido por el autor.

28 HäвERLE, op. cit., I69.

29 La interpretación es la misma exigida con relación al examen del caso concreto, como apunta HäBERLE, op. cit., I 73 .

30 Ibid., $\mathrm{I} 7 \mathrm{O}$.

3I Ibid., I73.

32 La idea de progreso aquí analizada guarda afinidad con la actividad de estabilización, resultante de la admisión de la universalidad como idea reguladora, por apuntar al progreso de la especie, 
se ve establecida de forma rígida una preponderancia de la dimensión de configuración de derechos con relación a la actividad restrictiva. Justamente porque se identifica una sustitución de la idea de restricción por la idea de límite. Como bien apunta Alexy, ello es así porque "cuando algo es restrictivo desde algún punto de vista, sigue siendo restrictivo aun cuando desde algún otro punto de vista sea configurador"33.

De allí el rechazo, justificado por Alexy, a la adopción expresa de una teoría interna de restricción de los derechos fundamentales, como lo defiende Häberle a la luz de la doctrina de Wolfgang Siebert ${ }^{34}$. Para Häberle, la teoría interna de restricción de los derechos en relación con el instituto del abuso del derecho -lo que permitiría, en términos civiles, la conexión con la delimitación del ejercicio de los derechos individuales- explica la relación del concepto de límite con la idea de contenido de un derecho: "El objetivo de la teoría interna es ver en una unidad el contenido y los límites de un derecho"35. La idea de límite al ejercicio de un derecho, íntimamente identificada con el contenido y con la esencia de ese derecho, apenas permite, en Häberle, reforzar la idea de que el desafío argumentativo en cada caso nuevo es prácticamente inviable en términos jurídicos. Una vez que el derecho sigue reafirmándose institucionalmente -como una finalidad propia del sistema- a cada estudio particular propuesto al análisis del intérprete, cualquier función de progreso efectivamente conectado a un ideal de libertad individual sigue comprometida por la adopción de una teoría interna de restricciones de los derechos. $\mathrm{Y}$ esto es así porque, o hay un compromiso individual respecto de lo que queda conformado legislativamente, o no hay de-

no al progreso individual. Sin embargo, debe haber en la función de progreso también un dato abstracto de corrección a lo estable, en la misma medida en que se predispone en la normatividad -aun en abstracto- una corrección de rumbos desde el discurso real propuesto en concreto. Eso ocurre no porque el progreso, en esta hipótesis, dependa, de forma casuística, de un determinado hecho concreto para imponerse como idea reguladora -si esta ya está presupuesta-. Ocurre, más bien, porque el enunciado dogmático se ocupa tanto de la tarea de fundamentación realizada en la actividad legislativa, como de los valores en permanente cambio en la sociedad. Es lo que sostiene Alexy al afirmar que "la ampliación de la discusión jurídica en la dimensión temporal, objetiva y personal, hace posible ofrecer comprobaciones y diferenciar los enunciados dogmáticos en una medida considerablemente mayor de lo que sería posible en discusiones que se desarrollan en forma puntual. Con ello se hace posible algo así como un progreso de la dogmática” (Alexy, Teoría de la argumentación jurídica, cit., 257).

33 Alexy, Teoría de los derechos fundamentales, cit., 323.

34 En el análisis de la conformación de derechos subjetivos, con alcances específicos en el ámbito del derecho civil, Siebert defiende la adopción de una teoría interna de restricciones a los derechos fundamentales entre 1934 y I935, contribuyendo, al mismo tiempo, como profesor en la Alemania nazi y siendo identificado con la Escuela de Kiel, juntamente con Karl Larenz, Franz Wieacker, Ernst Rudolf Huber, Georg Dahm y Frederich Schaffstein (Blanc, C. A., "La fundamentación teórica del Terror de Estado en la filosofía jurídica nacionalsocialista de Karl Larenz", AFD (Anuario de Filosofía del Derecho), a. xxx, Madrid, CPAGr, 20 I4, I I9-I37).

Häвerle, P., La garantía del contenido esencial de los derechos fundamentales, cit., I67. 
recho: "Cualquier delimitación de un derecho fundamental por el legislador es simultáneamente material, determinación puntual del contenido del derecho" ${ }^{6}$.

\section{La concepción estrecha de la configuración de derechos en Alexy}

En un sentido distinto procede el empeño de Alexy por la adopción de una teoría externa de restricciones de los derechos fundamentales ${ }^{37}$. El autor, partiendo de una visión discursiva del sistema jurídico, orientada por una interpretación fundada en argumentos jurídicos y en bases racionales, con pretensión de corrección, sostiene que la tarea de la dogmática asume tres compromisos centra$\operatorname{les}^{38}$ : (i) el análisis lógico de los conceptos jurídicos; (ii) la reconducción de este análisis a un sistema que opere por medio de enunciados, conectando fuentes jurídicas; y (iii) la aplicación de los resultados de este análisis en la fundamentación de las decisiones jurídicas, tengan ellas una finalidad de alcance ideal y abstracto (universal) o inclusive real y concreto (particular). Sin embargo, ello no reduce la tarea del intérprete a un trabajo puramente analítico ${ }^{39}$.

Existe, en la definición de Alexy, el compromiso asumido por el intérprete de que toda y cualquier interpretación en el derecho tenga un alcance institucional y, simultáneamente, una preocupación por viabilizar la corrección de rumbos en la adopción de soluciones jurídicas de conflictos reales. Algo que se desdobla en funciones propias del ejercicio de la interpretación por medio del análisis de las funciones de estabilización, progreso, descarga, técnica, control y heurística de la propia dogmática ${ }^{\circ}$.

En ese panorama, hay efectivamente una doble dimensión de análisis cuando se trata del tema de la restricción de los derechos. Alexy identifica un presupuesto de libertad máxima del individuo en su actuar público. El control por parte del derecho es, por lo tanto, externo, siempre que se identifique una situación de conflicto suficiente y necesario para promover esta intervención institucional. Esto explica la relevancia del problema de las restricciones de los derechos fundamentales.

$36 \mathrm{Ibid.,}$ I67. Y aquí se puede identificar la fuerza que la interpretación en abstracto asume en la doctrina de Häberle, al punto de determinar, a priori, el contenido del derecho.

37 La defensa de una teoría externa en Alexy está ligada a la preocupación del autor de conectar la interpretación del legislador a la interpretación para el caso por medio de argumentos de interpretación. Ver Vidal, I. L., "Interpretación y aplicación del derecho”, en Lagier, D. G. (coord.), Conceptos básicos del derecho, Madrid, Marcial Pons, 20 I 5, I 94-I 99.

$3^{8}$ Alexy, R., Teoría de la argumentación jurídica, Madrid, CEPC, 20 I 2, 243.

39 "Una dogmática del Derecho es una serie de enunciados que se refieren a las normas establecidas y a la aplicación del Derecho, pero no pueden identificarse con su descripción, están entre sí en una relación de coherencia mutua, se forman y discuten en el marco de una ciencia jurídica que funciona institucionalmente y tiene contenido normativo". AleXY, R., op. cit., 246.

40 Ibid., 255. 
Para dilucidar el alcance de la doble dimensión restrictiva de los derechos fundamentales por el derecho -como configuración y restricción propiamente dicha-, parte el autor de algunos presupuestos específicos de análisis. En primer lugar, el presupuesto de que el concepto de restricción comprende dos particularidades bastante distintas: el derecho y sus restricciones. Esto significa reconocer que hay dos momentos de identificación de un derecho. El momento del derecho en sí mismo, sin ningún tipo de restricción, y el momento en el que el derecho, por circunstancias especiales y justificadas, puede sufrir restricciones. Este, para Alexy, es el "derecho restringido" ${ }^{1}$. En consecuencia, el mismo examen con relación a la norma iusfundamental conduce a concluir que no toda norma de derecho ordinario que tenga por objeto una situación de interés de un derecho fundamental resulta en una restricción a los derechos. Es posible que se trate de una norma que simplemente tenga por finalidad la configuración de un derecho ${ }^{42}$.

Ejemplo claro de tal construcción normativa en el ordenamiento jurídico brasileño es el del enunciado del artículo 2 I C. C., que en su primera parte determina que "la vida privada de la persona natural es inviolable". No hay ninguna indicación de restricción al derecho fundamental igualmente positivizado en el artículo $5 \cdot^{\circ}$, inciso $\mathrm{x}$, de la Constitución Federal brasileña, cuando se refiere a la protección a la intimidad y a la vida privada de la persona natural en términos abstractos. Por el contrario, lo que establece la norma infraconstitucional es una configuración, en el ámbito de las relaciones entre privados -por lo tanto, facilitando, a priori, la interpretación para el caso-, de la garantía de un derecho fundamental positivizado en la Constitución 43 . El artículo referido difiere en el enunciado de la primera parte del artículo 20 C. C., cuya redacción ofrece una norma de restricción legislativa a la garantía constitucional del mismo artículo $5^{\circ}$, inciso $\mathrm{x}$, de la Constitución Federal, específicamente con relación a la protección del honor y la imagen de las personas.

Un segundo presupuesto se refiere al énfasis del alcance de un concepto de configuración de derechos. Para Alexy, una concepción estrecha del concepto de conformación de derechos es posible si la intención es la de potencializar la libertad del individuo en sociedad para que desarrolle efectivamente su autonomía ${ }^{44}$. En contrapartida, debe concebirse un concepto amplio de restricción,

4I Ibid., 268.

42 Ibid., 322.

43 Para un amplio desarrollo del tema en el derecho civil brasileño, Cachapuz, M. C., Intimidade e vida privada no novo Código Civil brasileiro, Porto Alegre, Fabris Editor, 2006.

44 Cuando se habla de autonomía, se está, en verdad, discutiendo una cuestión más amplia que la presupuesta en un derecho general de libertad por el individuo. Habermas establece una distinción bastante clara al respecto: los conceptos se diferencian por el ámbito de su extensión. Mientras la libertad es siempre subjetiva, por estar fundada en las peculiaridades del individuo -sus "máximas de prudencia, por las preferencias o motivos racionales"-, la autonomía es un 
posibilitando el permanente desafío a lo que está determinado en el sistema jurídico.

Hay una proporcionalidad en tal construcción también en términos abstractos. Cuando se está frente a un concepto estrecho de conformación y un concepto amplio de restricción de derechos, el equilibrio en el ejercicio de argumentación es alcanzado por el funcionamiento del propio sistema jurídico y de las funciones exigidas a los enunciados dogmáticos. O sea, aquello que se ve configurado como un derecho no necesita justificación. La restricción al derecho, de otra parte, tanto en abstracto como en concreto, exige argumentación necesaria y suficiente para el desafío a la garantía iusfundamental, aunque sea para la creación de una norma por la vía ordinaria45. Esta es la distinción esencial para la adopción de una teoría externa de restricción de los derechos fundamentales.

Solo una concepción estrecha del concepto de conformación, a la que corresponde un adecuadamente amplio concepto de restricción, responde a las exigencias de racionalidad de la fundamentación iusfundamental. Aquello que es calificado como configuración no necesita ser fundamentado frente al derecho fundamental. Al mantenerse amplio el ámbito de lo que requiere y es susceptible de justificación, mediante un concepto estrecho de configuración, no se asegura todavía, por cierto, que solo habrán de llevarse a cabo restricciones permitidas, pero sí que ninguna restricción podrá liberarse de la fundamentación; ello es un presupuesto esencial para que solo se normen restricciones permitidas ${ }^{46}$.

concepto que presupone una estructura de intersubjetividad, determinado por máximas aprobadas por la prueba de la universalización. Según dicho autor, "en cuanto a la libertad subjetiva, no es difícil imaginar que algunas personas puedan gozar de la libertad y otras no, o que algunas personas puedan ser más libres que otras. La autonomía, por el contrario, no es un concepto distributivo y no puede ser alcanzado individualmente. En tal sentido enfático, una persona solo puede ser libre si todas las demás lo son igualmente. La idea que quiero subrayar es la siguiente: con su noción de autonomía, Kant ya introduce un concepto que solo puede explicarse plenamente dentro de una estructura intersubjetiva" (Habermas, J., O futuro da natureza bumana, São Paulo, Martins Fontes, 2004, I3).

45 La relación establecida por Alexy, en términos restrictivos es también comprendida en la doctrina de Carlos Bernal Pulido a partir de una aplicación del principio de proporcionalidad. Es decir que "en el Estado constitucional no puede valer cualquier restricción a los derechos fundamentales sino sólo aquellas restricciones que sean: idóneas para contribuir a la obtención de cierto fin legítimo; necesarias, es decir, las más benignas entre los medios alternativos que gocen de por lo menos la misma idoneidad para conseguir la finalidad deseada; y proporcionales en sentido estricto, es decir, aquellas que logren un equilibrio entre los beneficios que su implementación representa y los perjuicios que ella produce. De esta manera, el principio de proporcionalidad es la restricción de la restricción, el límite de los límites a los derechos fundamentales, el criterio que condiciona la validez de los límites que el Estado impone a los derechos fundamentales" (Bernal Pulido, C., El derecho de los derechos, Bogotá, Universidad Externado de Colombia, 2005, 82).

46 Alexy, R., Teoría de los derechos fundamentales, Madrid, CEPc, 200 I, 329. 
Tales consideraciones acerca del ámbito de actuación de las configuraciones y restricciones de los derechos permiten, además, cuando es propuesto el examen del caso concreto, que se identifique que toda solución extraída de una ponderación frente a un conflicto de derechos fundamentales sea comprendida como el producto de una restricción en concreto, y no como la consecuencia de una subsunción a la configuración, en abstracto, de un derecho previamente definido en su contenido. De ello se extrae, en términos interpretativos y de manera coherente con la pretensión de reconocimiento de una dimensión institucional del sistema jurídico, la regla establecida para el caso. Esta, como resultado de una ponderación particular, no necesariamente configura un enunciado dogmático suficiente para que sea utilizada genéricamente como regla para toda y cualquier situación de conducta en términos análogos. Pero permite, por la propia analogía, que sea utilizada para el establecimiento de un par de comparación en términos interpretativos, por un ejercicio propio de ponderación ${ }^{47}$.

No se desestima, además, la misma racionalidad, para la prueba de la universalidad, pues la restricción está dirigida al legislador, y no al juez. El mismo compromiso de descarga de argumentos con relación al desafío de la protección de derechos generales de libertad e igualdad es exigido al intérprete en abstracto, en caso de que, en la creación de una ley infraconstitucional, quede evidenciada una justa restricción al derecho fundamental ${ }^{4}$.

El tercer presupuesto, resultante de los anteriores y relativo a la distinción entre configuración y restricción, que ha de ser tenido en cuenta es: el hecho de que el carácter de razonabilidad acogido para la edición de una norma y para la tipificación de una conducta (por el establecimiento de una prohibición, de un hacer obligatorio o por el establecimiento de competencias que alcancen el ejercicio de posiciones jurídicas), no aleja la dimensión restrictiva de esta misma norma, cuando involucre un derecho general de libertad o de igualdad de la per-

47 Para Alexy, la analogía no debe ser despreciada como forma de argumento; sin embargo, puesto que se basa en principios de universalidad y de igualdad, exige que se considere la dificultad típica de aplicación de un principio de igualdad: "El establecimiento de la semejanza jurídicamente relevante no puede surgir únicamente de semejante análisis [...] presupone valoración. Para la fundamentación de esta valoración son admisibles todos los argumentos posibles en el discurso jurídico" (Alexy, Teoría de la argumentación jurídica, cit., 269). Esto contesta, en parte, a la indagación acerca del papel y de la fuerza de la jurisprudencia, en la medida en que se exige una prueba más amplia de racionalidad -y, por lo tanto, no fundada en las peculiaridades de una única decisión jurídica en términos de interpretación- para la descarga de argumentos cuando es desafiada la configuración específica de un derecho. Es lo que normalmente ocurre cuando existen un interés en conflicto y derechos fundamentales en concreto que han de ser ponderados.

48 "El criterio de no obstaculización de la realización del principio iusfundamental implica que siempre que sea necesaria (caso de derecho fundamental actual) o meramente posible (caso de derecho fundamental potencial) una ponderación orientada por el principio de proporcionalidad, no debe suponerse una configuración, sino una restricción”: Alexy, R., Teoría de los derechos fundamentales, Madrid, CEPC, 200I, 328. 
sona ${ }^{49}$. Aquí, el examen de la fundamentación en Alexy exige un nivel de detalle más amplio para evitar equívocos.

La preocupación de Alexy se centra en la adopción de una teoría interna de restricciones de derechos fundamentales: la constatación de que cualquier limitación de un derecho fundamental debe ser vista como parte de la determinación de su contenido. Según Häberle, "el legislador que limita los derechos fundamentales también los determina con ello en cuanto a su contenido y al revés"50.

La fragilidad de tal construcción -aunque limitada a la perspectiva de análisis de la interpretación por el legislador- quedó referida gracias a la crítica de Alexy, en el sentido de que para la adopción de una teoría interna sería siempre necesario un condicionamiento del examen de cualquier restricción a la hipótesis de abuso de un derecho, puesto que presupone un determinado contenido configurador de un derecho por parte del sistema jurídico. Tal situación, aparentemente, permite al intérprete una relativización de la concepción material de un derecho cuando se trata de analizar un caso concreto -aunque el ejercicio de interpretación se torne bastante difícil, por la necesidad, en la misma medida, de la configuración previa del propio concepto de abuso de derecho-. Pero se trata de una situación de construcción argumentativa extremadamente difícil cuando el problema es de un supuesto abuso por parte del legislador en la configuración del tipo normativo, al editar una norma restrictiva de un derecho fundamental en el ámbito infraconstitucional.

Debe distinguirse: una realidad es la de las normas de configuración de institutos del derecho civil -generando competencias específicas para el caso de capacidad, matrimonio, propiedad, establecimiento de sociedad-, en los que hay una confirmación de determinadas garantías constitucionales iusfundamentales. En esas hipótesis, es posible que se vean establecidas situaciones de conformación de derechos, para garantizar, justamente, la titularidad de determinadas posiciones jurídicas. Otra circunstancia es la de las normas que, estableciendo prohibiciones o determinaciones de un hacer específico, generen restricciones al ejercicio de libertades. Aunque sean necesarias tales restricciones, no se reconoce en la libertad residual -que es el producto de interpretación en abstracto del legislador- la configuración de un derecho.

\section{El sistema de posiciones jurídicas como un sistema de posiciones jurídicas fundamentales}

Situación análoga al tema de las restricciones y configuraciones de derechos es la del análisis de las posiciones jurídicas fundamentales que pueden ser iden-

49 Alexy, R., Teoría de los derechos fundamentales, cit., 324.

50 HäBerLe, P., La garantía del contenido esencial de los derechos fundamentales, Madrid, Dykinson, $2003,167$. 
tificadas en el sistema jurídico cuando los derechos fundamentales son observados como derechos subjetivos. Esto se da cuando se conectan los derechos fundamentales positivizados en el ordenamiento jurídico con el ejercicio de los derechos por sus titulares, a partir de un enfoque analítico ${ }^{5}$, de forma tal que se identifiquen situaciones específicas de ejercicio de derecho o de sujeción de las personas a determinadas restricciones. La conexión parece resultar de la forma como los individuos se vinculan en el espacio público, asumiendo posiciones e intereses diversos, en la medida en que se conectan al ejercicio de determinados derechos y, en esa medida, le otorgan determinado contenido.

Según Alexy, son tres las concepciones posibles para la comprensión de la estructura de los derechos subjetivos: (a) los derechos a algo, (b) las libertades y (c) las competencias.

Cuando se refiere a derechos a algo, Alexy se preocupa por una relación entre titulares de derechos y deberes a partir de la estructuración de una relación jurídica en la que hay, claramente, una preocupación por el objeto del derecho en cuestión siendo "siempre una acción del destinatario" ${ }^{2}$. De forma directa, al tra$\operatorname{tar}$ los derechos a algo, Alexy los asocia a la estructura ofrecida por W. N. Hohfeld en la definición de opuestos y correlativos jurídicos de posiciones jurídicas entre deberes y derechos, aunque acentúe determinadas diferencias específicas ${ }^{53}$. En la estructura de la idea de derechos a algo reconoce una posición jurídica fuerte con relación a la realización del objeto pretendido, independientemente de que se trate de una relación entre privados o de una relación entre un particular y el Estado, en lo que podría ser identificado, con relación al titular del derecho, como un "derecho de defensa" - cuando se trate de un derecho a una acción negativa de alguien o del Estado, capaz de abarcar: (i) el derecho de no impedir acciones individuales; (ii) el derecho a la no afectación de propiedades y situaciones de garantías individuales; y (iii) el derecho a la no eliminación de posiciones jurídicas- o como un "derecho a una pretensión" -en la realización de derechos

$5^{\mathrm{I}}$ En el análisis de los derechos fundamentales como derechos subjetivos, Alexy sostiene la posibilidad de establecer un modelo de tres niveles de conceptualización de derechos subjetivos. Los derechos pueden ser vistos: en un enfoque normativo, como correspondientes a las razones ofrecidas para saber por qué alguien es titular de un derecho y qué derecho es este -algo que aproxima la interpretación de los derechos subjetivos a una visión más positivista del derecho-; en una perspectiva empírica, por el abordaje de análisis de los derechos subjetivos en relación con argumentos históricos y teleológicos - por lo tanto, con aproximaciones a una visión más funcionalista y sociológica del derecho-; y, por fin, en un enfoque analítico, en el que se adopta como elementos constitutivos del concepto de derecho tanto el fin práctico alcanzado por determinado derecho subjetivo -"es decir, la utilidad, ventaja, ganancia, que ha de ser proporcionada por el derecho" (Alexy, Teoría de los derechos fundamentales, cit., I 79)- como la protección ofrecida por este derecho en el sistema jurídico. La aproximación, en tal caso, es a una doctrina de carácter discursivo.

52 Alexy, R., Teoría de los derechos fundamentales, cit., I 87.

53 Ibid., 202. 
de acciones positivas, tanto en relación con prestaciones fácticas como con prestaciones normativas ${ }^{54}$.

Otra concepción de derecho subjetivo, para Alexy, se relaciona con una prerrogativa de libertad. En la estructura analítica referida, hay una relación entre el titular de una libertad -o de la negativa de esta-, un impedimento a esta libertad y un objeto de libertad. En tal hipótesis, el objeto de una libertad es siempre una "alternativa de acción" 55 porque se presupone la posibilidad amplia de una libertad negativa. El objeto de una libertad, por lo tanto, permite apenas una determinación no definitiva con respecto al ejercicio de la libertad presupuesta. Lo que se quiere confirmar con tal afirmación no es la circunstancia de ausencia de libertad, sino la consideración de que, siendo esta presupuesta, la libertad jurídica es siempre correspondiente a un deber opuesto de cumplimiento.

En Hohfeld esta relación jurídica es explicada a partir de un modelo de opuestos jurídicos donde, en la misma proporción que se establece una relación entre libertad y deber, se asocia una proporcionalidad entre derecho subjetivo y no derecho. Corresponde que a una libertad -traducida por Hohfeld como un privilegio $5^{6}$ - se correlaciona un no derecho, y a un derecho subjetivo se correlaciona un deber57.

Un privilegio es el opuesto de un deber, y el correlativo de un no-derecho. En el ejemplo último, mientras que $\mathrm{x}$ tiene un derecho o pretensión a que $\mathrm{y}$ no entre en el inmueble del primero, $x$ tiene el privilegio de entrar en el inmueble; o, con otras palabras, $\mathrm{x}$ no tiene el deber de permanecer fuera del mismo. El privilegio de entrar en el inmueble es la negación del deber de permanecer fuera de él $5^{8}$.

No se puede dejar de identificar que entre los modelos de opuestos y los modelos de correlativos jurídicos, como los presentados por Hohfeld, existe una misma determinación de razones distintivas para la interpretación, al igual que ocurre con las razones ofrecidas por Alexy en la diferenciación entre principios y reglas. Cuando se parte de un modelo de opuestos jurídicos -como entre derecho y no derecho, libertad y deber59-, hay una determinación prima facie del derecho en discusión. Es diferente de la asociación que pueda hacerse desde modelos de correlativos jurídicos -como entre derecho y deber, libertad y no derecho-. En estos, la determinación parte de una razón definitiva, como al nivel de las reglas, justamente porque presupone una descarga de argumentos distintos con relación a

Ibid., I89-194.

Ibid., 2 I 4 .

56 Hohfeld, W. N., Conceptos jurídicos fundamentales, México, Fontamara, 2009, 53.

57 Ibid., 49.

58 Ibid., 53 .

59 Ibid., 49 . 
la posición jurídica de donde se parte -en la correlación de derechos y deberes, de libertades y no derechos-. Se presupone, desde el punto de vista formal, que esta correlación de posiciones jurídicas deba corresponder a una universalidad ideal de conductas. Esto no evita que, evidenciado el conflicto concreto, ocurra un desafío de la situación de igualdad o de libertad presupuesta en el modelo de correlación - p. ej., cuando se cuestiona la medida de la autonomía determinante en la declaración del contratante para el establecimiento de una cláusula contractual reconocida como abusiva-. Desde luego, el desafío propuesto permite comprender la necesidad de adopción de un modelo de opuestos jurídicos, conduciendo la interpretación hacia una determinación no definitiva con relación a la conformación de un derecho.

Pero hay todavía una tercera concepción posible del reconocimiento de un derecho subjetivo que corresponde a las competencias, esto es, desde la idea de ejercicio de un poder o, más específicamente, de un poder jurídico. La aproximación aquí ofrecida tiene que ver con la posibilidad de modificar una situación jurídica del individuo por la práctica de determinadas acciones que conduzcan a una posición jurídica reconocida institucionalmente. Lo que se reconoce es la prerrogativa de que, a partir de un conjunto de actos, se otorgue a los individuos, por medio de un previo establecimiento institucional -fáctico o normativo-, la titularidad de un derecho que, en principio, no posee por naturaleza.

Hohfeld igualmente trató el tema de las competencias con relación a la idea de derecho, al establecer parámetros de correlación o de oposición entre posiciones jurídicas resultantes de una competencia o de ausencia de una competencia (una no competencia) y de una inmunidad (no sujeción) o de una sujeción. No se limitó, por lo tanto, al sistema de posiciones jurídicas fundamentales de Alexy, sino que amplió el cuadro de variables disponibles para posibilitar la identificación de un sistema de tablas comparativas de correlativos y de opuestos jurídicos. O sea, desde la perspectiva de posiciones jurídicas correlacionadas, competencia y sujeción guardan la misma relación que hay entre derecho subjetivo y deber, así como la ausencia de competencia genera una situación de inmunidad genérica a la observancia de titularidad de una determinada posición jurídica institucional por parte de un tercero, tal como se identifica entre el ejercicio de una libertad y la observancia contraria de un no derecho; desde la perspectiva de posiciones jurídicas opuestas, una competencia y una ausencia de competencia guardan la misma relación que la idea de un derecho en oposición a un no derecho. Lo mismo se observa entre la inmunidad genérica a la observancia de titularidad de una determinada posición jurídica institucional por parte de un tercero y una sujeción a la titularidad del tercero con relación a la situación opuesta de ejercicio de una libertad y el deber contrapuesto (la libertad negativa).

$\mathrm{Al}$ establecer un cuadro de visualización de concepciones semejantes a derechos y deberes desde el ejercicio de posiciones jurídicas en el actuar público, Hohfeld parece haber ofrecido un esquema analítico bastante amplio, que per- 
mite una explicación lógica de la dinámica de manifestación del fenómeno jurídico para cualquier relación del derecho. $\mathrm{Y}$ esto es así porque parte tanto de una idea de derecho a algo como de las ideas de libertad, de competencia (poder) y de inmunidad (no sujeción).

\section{Configuración y restricción de derechos}

Si se considera correcta la prueba analítica propuesta por Hohfeld al sistema jurídico, permitiendo que se puedan sustituir las variables de condicionamiento a la manifestación de posiciones jurídicas ejercidas por los sujetos de derecho, la idea es que se pueda igualmente confirmar si existe racionalidad en la construcción de una interpretación -entonces relacionada con la solución concreta de casos propuestos a discusión jurídica- que sostenga que la concepción de derecho deba, necesariamente, partir de una restricción externa o de una restricción interna a los derechos fundamentales. En parte, Alexy propuso un test similar, al analizar, a partir de la concepción de derecho como una competencia de carácter estricto $-y$ no como un comportamiento resultante del establecimiento de una competencia-, la distinción entre las dimensiones de ilicitud y de validez de un acto jurídico ${ }^{60}$.

La comparación que ahora se propone está dirigida al tema de las restricciones y configuraciones de derechos subjetivos. Tratándose de una relación bidimensional -de distinción de dimensiones de restricción y de configuración de derechos-, la hipótesis sería la de probar en qué medida las relaciones de oposición y de correlación de posiciones jurídicas, correspondientes a derechos y deberes, pueden ser sustituidas por situaciones de restricción y de configuración de posiciones jurídicas, respectivamente.

Una pista inicial es ofrecida por Alexy cuando aclara respecto de los bienes que pueden sufrir restricciones: "Restringibles son bienes iusfundamentalmente protegidos (libertades/situaciones/posiciones de derecho ordinario) y posiciones prima facie concedidas por principios iusfundamentales" "I. Lo que quiere decir es que, cuando no exista relación directa con una proposición iusfundamental, no se habla de restricciones a derechos: "Las normas que no tienen nada que ver con un derecho fundamental no son con respecto a este derecho fundamental normas restrictivas y tampoco normas configuradoras" ${ }^{62}$. Pero siempre que

60 Alexy, R., Teoría de los derechos fundamentales, cit., 233. Para una distinción de las concepciones de validez y nulidad frente al problema de la validez constitutiva (o existencia) y de la validez regulativa, ver Alemany, M., "Validez y nulidad", en Lagier, D. G. (coord.), Conceptos básicos del derecho, Madrid, Marcial Pons, 2015, I 75-184. En el derecho brasileño, ver Vilanova, L., Causalidade e relação no direito, $5 .^{\text {a }}$ ed., São Paulo, Noeses, 2015.

6 I Alexy, R., Teoría de los derechos fundamentales, cit., 272.

62 Ibid., 322. 
exista un contenido jurídico relacionado con derechos fundamentales es posible hablar de restricción de los derechos.

A partir de tal comprensión -y ahora principalmente en el campo de la interpretación para el caso concreto- se podría sostener que cuando se toma en cuenta una situación no restrictiva de un derecho fundamental -porque no se promueve una discusión jurídica sobre restricción a una libertad-, la aproximación de una situación de análisis de las posiciones jurídicas en juego a partir de correlativos jurídicos de la tabla de Hohfeld debe ser promovida. En estos casos, se parte de una configuración a priori del ejercicio de posiciones jurídicas correspondientes y que, por lo tanto, presuponen una determinada configuración aceptada y deseada por los individuos. Son situaciones que, observada la característica del caso, no se encuentran desafiadas por una restricción de ámbito iusfundamental. Se trata de comprender la configuración preliminar de determinadas situaciones fácticas y jurídicas aceptadas por los individuos -lo que es típico en el derecho civil, como en la hipótesis de una contratación establecida entre las personas-, y partir de tal perspectiva - a priori, por una razón definitiva- para la interpretación del caso. Es, por lo tanto, una situación que no exige más que la adopción de una teoría interna de restricciones de los derechos subjetivos.

Esto es distinto de la situación que debe ser presupuesta cuando hay una propuesta de interpretación jurídica en términos de análisis de restricciones de los derechos fundamentales, a partir de la ocurrencia de un conflicto entre bienes iusfundamentalmente protegidos. Allí, siguiendo a Alexy, justamente porque se parte de la concepción de que siempre hay restricción de derechos cuando se habla de limitación de derechos fundamentales, la aproximación posible es a partir de la tabla de opuestos jurídicos. O sea, cuando se dice que alguien tiene derecho a algo es porque, en oposición, la otra parte sufrirá una restricción, por un derecho a no algo -un no-derecho-. El ejemplo aplicado por Alexy se refiere a los modelos de normas de mandato y de prohibición, en el que se visualiza la hipótesis en la relación del sujeto de derecho con el Estado. Pero lo mismo se podría aplicar con relación a los conflictos de libertades entre particulares.

En derecho civil, cuando se discute una limitación a la autonomía del individuo se tratan las posiciones jurídicas en oposición, porque el discurso propuesto ocurre en términos de restricción a una libertad de uno de los contratantes. En tales casos, el análisis no puede partir de la misma comprensión de interpretación por una teoría interna de restricción a los derechos, justamente porque la discusión propuesta se refiere a la configuración de derechos y deberes establecida a priori entre las partes contratantes y sobre la posibilidad de su desafío en concreto. La interpretación, por lo tanto, debe adoptar una teoría externa de restricción de los derechos.

Es posible que se reconozca que entre posiciones jurídicas fundamentales y restricciones a los derechos subjetivos existan conexiones bastante más estrechas. Según Alexy, al menos en términos iusfundamentales, la respuesta más simple 
está en el hecho de que "un derecho fundamental como un todo es un haz de posiciones iusfundamentales" ${ }_{3}$. Adaptándose el mismo ejercicio interpretativo para toda y cualquier relación jurídica -incluyéndose, principalmente, las relaciones de derecho civil de forma general y los reflejos de concepciones iusfundamentales en el ámbito de las relaciones civiles entre privados-, es posible que se extraiga la comprensión de que un derecho subjetivo, en su totalidad, es un haz de posiciones jurídicas fundamentales. En tal medida, es la identificación de una determinada posición jurídica, en perspectiva de correlación o de oposición a una situación de ventaja (un derecho, una libertad, una competencia o una inmunidad) o desventaja jurídica (un deber, un no derecho, una no competencia o una sujeción), siguiendo el modelo de Hohfeld, lo que permitirá identificar, frente a la condición concreta propuesta, la hipótesis de análisis configurativa o restrictiva de un derecho subjetivo en concreto.

Así, para el examen del caso concreto, cuando se trate de una discusión jurídica que exija valorar posiciones fundadas en opuestos jurídicos, la discusión propuesta corresponderá, prima facie, a una situación de restricción del derecho subjetivo por aplicación de una teoría externa de restricción de los derechos; tratándose de un examen de posiciones jurídicas basado en correlativos jurídicos -y siempre y cuando no exista una discusión preliminar entre libertades o situaciones de igualdad en juego-, corresponderá, a priori, a una situación de configuración de un determinado derecho subjetivo. Esto exigirá, en el caso, la aplicación de una teoría interna de restricciones de los derechos, puesto que la interpretación está orientada por un acuerdo previo con relación a la conformación de determinado derecho.

\section{Conclusiones}

En resumen, el presente estudio se ocupa del problema relativo al análisis del contenido de los derechos subjetivos y a la configuración y restricción de estos. Parte de la constatación de que el derecho civil, por integrar el sistema jurídico, sufre los efectos de las normas iusfundamentales de forma genérica. La materia interesa en la medida en que permite afrontar el problema de las limitaciones y de la ponderación de los derechos subjetivos, tanto en una dimensión institucional como en una relación específica y particular de determinada situación concreta de conflicto de intereses.

Häberle defiende la teoría de que los derechos fundamentales comparten un doble carácter en su estructura: presentan tanto una dimensión institucional-objetiva como una dimensión jurídico-individual, otorgando a sus titulares derechos públicos subjetivos. La lógica de Häberle es que toda y cualquier restricción de los derechos fundamentales está necesariamente relacionada con 
una concepción amplia de configuración de esos mismos derechos. Por su parte, Alexy adopta una teoría externa de restricciones de los derechos fundamentales, partiendo de una visión discursiva del sistema jurídico, orientada por una interpretación fundada en argumentos jurídicos y en bases racionales, con pretensión de corrección. Como presupuesto de la interpretación, se encuentra la comprensión de que el concepto de restricción tiene dos particularidades distintas: el derecho y sus restricciones.

Situación semejante se presenta con el análisis de las posiciones jurídicas fundamentales que pueden ser identificadas en el sistema jurídico. La conexión resulta de la forma como los individuos se vinculan en el espacio público, asumiendo posiciones e intereses diversos en el ejercicio de derechos y deberes, libertades y no derechos. La comparación que se propone, consecuentemente, es la de probar el sistema analítico de posiciones jurídicas fundamentales, como en el modelo de Hohfeld, buscando justificar, racionalmente, la aplicación de las teorías interna o externa de restricción de los derechos subjetivos. Es lo que se propone como la comprensión de que un derecho subjetivo, visto de forma amplia, es un haz de posiciones jurídicas fundamentales.

En ese sentido, es la identificación de una determinada posición jurídica, en perspectiva de correlación o de oposición a una situación de ventaja (un derecho, una libertad, una competencia o una inmunidad) o desventaja jurídica (un deber, un no derecho, una no competencia o una sujeción), lo que permitirá identificar, frente a la condición concreta propuesta, hipótesis de análisis de una restricción -debido a la adopción de una teoría externa- o de una configuración -en el caso de la adopción de una teoría interna- de un derecho subjetivo.

\section{Bibliografía}

Alemany, M., "Validez y nulidad", en Lagier, D. G. (coord.), Conceptos básicos del derecho, Madrid, Marcial Pons, 2015.

Alemany, M., Teoría de la argumentación jurídica, Madrid, CEPc, 20 I 2.

Alemany, M., Teoría de los derechos fundamentales, Madrid, CePc, 200 I.

Blanc, C. A., "La fundamentación teórica del Terror de Estado en la filosofía jurídica nacionalsocialista de Karl Larenz", AFD (Anuario de Filosofía del Derecho), a. xxx, Madrid, CPAGR, 2014.

Carrió, Genaro. "Nota preliminar”, en Hohfeld, W. N., Conceptos jurídicos fundamentales, México, Fontamara, 2009. 
Cachapuz, M. C., Intimidade e vida privada no novo Código Civil brasileiro, Porto Alegre, Fabris Editor, 2006.

Guastini, R. Distinguiendo. Estudios de teoría y metateoría del derecho, Barcelona, Gedisa, I999.

HäвERLE, P., La garantía del contenido esencial de los derechos fundamentales, Madrid, Dykinson, 2003.

Habermas, J., O futuro da natureza bumana, São Paulo, Martins Fontes, 2004.

Hohfeld, W. N., Conceptos jurídicos fundamentales, México, Fontamara, 2009.

Moreso, J. J. y Vilajosana, J. M., Introducción a la teoría del derecho, Madrid, Marcial Pons, 2004.

Nipperdey, H. C. "Livre desenvolvimento da personalidade", en Dürig, G., Nipperdey, H. C. y Schwabe, J. Direitos fundamentais e direito privado. Textos clásicos, Porto Alegre, Sérgio Antônio Fabris Editor, 201 2, p. 76.

Pulido, C. B., El derecho de los derechos, Bogotá, Universidad Externado de Colombia, 2005 .

VIDAL, I. L., "Interpretación y aplicación del derecho”, en LAGIER, D. G. (coord.), Conceptos básicos del derecho, Madrid, Marcial Pons, 2015.

Vilanova, L., Causalidade e relação no direito, 5. ${ }^{\text {a }}$ ed., São Paulo, Noeses, 20 I 5. 